\title{
A metric for bounded response scales
}

\author{
PETER H. SCHÖNEMANN \\ Purdue University, West Lafayette, Indiana 47907
}

\begin{abstract}
It is suggested that bounded response scales may induce nonlinearities in psychophysical functions. This point is illustrated with an example of MDS, in which the intuitively plausible rationale of the city-block metric, which is based on ordinary addition of line segments, may conflict with the boundedness of the dissimilarity scale provided by the experimenter. On replacing ordinary addition with the addition rule for hyperbolic tangents, a metric intermediate between the city-block and the sup metrics is obtained that preserves the upper bound. In the one-dimensional case, it predicts overestimation for halving, underestimation for doubling, and an upward shift of the midpoint for middling.
\end{abstract}

The hyperbolic tangent function

$$
\begin{aligned}
\tanh (x)= & {[\exp (2 x)-1] /[\exp (2 x)+1], } \\
& -\infty<x<+\infty
\end{aligned}
$$

is an invertible map from the real open interval $(-\infty, \infty)$ onto $(-1,1)$. Its inverse,

$$
\tanh ^{-1}(y)=.5 \ln (1+y) /(1-y),-1<y<1,
$$

is familiar from statistics, in which it is used as a variancestabilizing transformation for sample correlations to obtain approximate tests under the nonnull case, $\mathrm{E}(\mathrm{r}) \neq$ 0 (Fisher's z transformation).

The hyperbolic tangent plays a central role in special relativity theory. There, it is used for expressing the law of addition of velocities, $\mathbf{v} \mathbf{i}$,

$$
\begin{gathered}
b_{12}=\left(b_{1}+b_{2}\right) /\left(1+b_{1} b_{2}\right), \\
b_{i}=v_{i} / c, c=\max \left(v_{i}\right),
\end{gathered}
$$

by an addition of hyperbolic tangents,

$$
\begin{aligned}
& \tanh \left(u_{1}+u_{2}\right)= \\
& \quad\left[\tanh \left(u_{1}\right)+\tanh \left(u_{2}\right)\right] /\left[\left(1+\tanh \left(u_{1}\right) \tanh \left(u_{2}\right)\right] .\right.
\end{aligned}
$$

As is well-known, the need for such a more general law over the simpler, Newtonian law

$$
\mathrm{v}_{12}=\mathrm{v}_{1}+\mathrm{v}_{2}
$$

arose from the observation that the speed of light, c, remains the same in all moving reference frames and, thus, constitutes an upper bound for all velocities $v_{i}$. Addition of two (rescaled) velocities $b_{1}$ and $b_{2}$ $(<1)$ by Equation 3 always leads to a composite velocity $b_{12}<1$.

Similar bounds may also arise in psychophysics, either as a consequence of certain anatomical or physiological limitations of the human transducer or as a consequence of the instructions imposed by the experimenter. An example of the first kind is the perception of speed, perhaps the most obvious analogy to the precedent from physics. Caelli, Hoffman, and Lindman (1978) transposed the basic ideas of relativity theory into this psychophysical setting. They showed how on direct measurement of the upper threshold for velocity perception, $c_{k}$ (where the subscript indicates that it may differ from subject to subject), so-called "Lorentz transformations" these thresholds and how these transformations can be used to predict subjective length contractions and time dilations, as they also arise in physics. On checking these predictions empirically, Caelli et al. found them confirmed.

An example of the second kind is multidimensional scaling (MDS), in which the subject is frequently asked to produce dissimilarity ratings on a bounded response scale ("How similar is jelly roll to peanut-butter sandwich on a scale from 0 to 9?"). Such data are typically analyzed with one of the Minkowski power metrics, most often the euclidean, with little if any concern for the fact that the observed ratings are per instruction bounded, whereas Minkowski power metrics are in principle unbounded.

This example will be pursued here in some detail to introduce the notion of a metric for bounded response scales in which the bounds may be imposed either by the experimenter or by the subject. These metrics may explain certain systematic nonlinearities in subjective spaces, as they have already been found in psychophysical experiments. The important one-dimensional case will fall out as a special case of the two-dimensional formulation.

\section{A METRIC FOR BOUNDED RESPONSE SCALES}

For notational convenience, let $d_{i j}=d_{i j} * / c *$ be the "relative dissimilarity" obtained by dividing the observed 
dissimilarities $\mathrm{d}_{\mathrm{ij}}$ * by a suitable upper bound.

$$
c^{*}=\max \left(d_{i j}^{*}\right)+e, e>0
$$

where the small positive constant e ensures that all relative distances $d_{i j}$ fit into the half-open interval $[0,1)$. In particular, this interval does not contain the upper bound $c=1$, which is "off limits," so to speak, to guarantee the existence of the inverse transformation (Equation 2). In practice, $c^{*}$ may be simply the largest admissible value of the response scale augmented by a small constant. In applications to psychophysical experiments involving one-dimensional continua, $\mathrm{c}^{*}$ may have to be determined empirically, as in the Caelli et al. (1978) study, in which it varied from subject to subject.

To return to the slightly more general two-dimensional example, in which the bounded response involves dissimilarity ratings of pairs of stimuli presumed to vary along two dimensions, let us assume the subject intends to employ the city-block (CB) metric

$$
d_{i j}=d_{i j 1}+d_{i j 2}, d_{i j 1}=\left|x_{i 1}-x_{j 1}\right|, d_{i j 1}=\left|x_{i 2}-x_{j 2}\right|,(7)
$$

in arriving at these ratings, but is aware that his response must never exceed the upper limit $c^{*}$ on the rating scale provided by the experimenter, no matter how close the two arguments, $\mathrm{d}_{\mathrm{ij} 1}{ }^{*}$ and $\mathrm{d}_{\mathrm{ij} 2}{ }^{*}$, are to this upper limit. The subject therefore has to contract his response in a continuous fashion, more so for larger than for smaller arguments. A concatenation that achieves this is the hyperbolic tangent addition (Equation 3). Thus, under this hypothesis, his metric for bounded response scales (MBR) will be

$$
\mathrm{d}_{\mathrm{ij}}=\left(\mathrm{d}_{\mathrm{ij} 1}+\mathrm{d}_{\mathrm{ij} 2}\right) /\left(1+\mathrm{d}_{\mathrm{ij} 1} \mathrm{~d}_{\mathrm{ij} 2}\right)=\tanh \left(\mathrm{u}_{\mathrm{ij} 1}+\mathrm{u}_{\mathrm{ij} 2}\right),
$$

where $d_{i j 1}=\tanh \left(u_{i j 1}\right)$ and $d_{i j 2}=\tanh \left(u_{i j 2}\right)$ are again the projections of the stimulus interval onto the two coordinate axes and $u_{\mathrm{ij} 1}$ and $\mathrm{u}_{\mathrm{ij} 2}$ are hyperbolic angles. For the concatenation of two segments in one dimension, one obtains by the same logic:

$$
d_{i j}=\left(d_{i k}+d_{k j}\right) /\left(1+d_{i k} d_{k j}\right)=\tanh \left(u_{i k}+u_{j k}\right),
$$

where $\mathrm{k}$ is any point between $\mathrm{i}$ and $\mathrm{j}$ on the onedimension continuum. Equivalently (after translating $\mathrm{k}$ to the origin), Equation 9 can be viewed as a concatenation rule for bounded norms.

The mapping (Equation 8) is nonnegative for nonnegative arguments and symmetric. To see that it also satisfies the triangle inequality, observe first that

$$
\begin{gathered}
a, b, c \geqslant 0, a+b \geqslant c \text { implies } \\
a /(a+1)+b /(b+1) \geqslant c /(c+1),
\end{gathered}
$$

because reversal of the inequality in the implication leads to a contradiction. The projections $d_{i j 1}$ onto the abscissa are by definition $\mathrm{CB}$ and, hence, satisfy the triangle inequality, as do the projections onto the ordinate. On adding the two inequalities,

$$
\mathrm{d}_{\mathrm{ij} 1}+\mathrm{d}_{\mathrm{jk} 1} \geqslant \mathrm{~d}_{\mathrm{ik} 1} \text { and } \mathrm{d}_{\mathrm{ij} 2}+\mathrm{d}_{\mathrm{jk} 2} \geqslant \mathrm{~d}_{\mathrm{ik} 2} \text {, }
$$

and using Equation 10, one obtains the triangle inequality for the MBR (Equation 8), which thus is a bona fide distance function. However, it is not a distance function with additive segments, because distances along a straight line will "add" up to a smaller value than they would with ordinary addition, as Equation 9 shows. This is simply a consequence of the intended effect that the result must never exceed the upper bound of 1 , no matter how close both arguments may be to this upper bound.

The MBR (Equation 8) and its special case (Equation 9) for one dimension imply a number of systematic distortions relative to the arguments $d_{i j 1}$ and $d_{i j 2}$ which can be checked empirically. For the one-dimensional case, one finds that Equation 9 implies for a doubling task with bounded response scales

$$
y=d(x)=2 x /\left(1+x^{2}\right), 0 \leqslant x<1,
$$

and for halving tasks with bounded scales

$$
x=h(y)=\left(1-\sqrt{1-y^{2}}\right) / y, 0 \leqslant y<1,
$$

and finally, for middling

$$
\begin{gathered}
x=m\left(y_{1}, y_{2}\right)=\left(1+y_{1} y_{2}-\sqrt{1-y_{1}^{2}} \sqrt{\left.1-y_{2}^{2}\right)} /\right. \\
\left(y_{1}+y_{2}\right), 0 \leqslant y_{1}<y_{2}<1 .
\end{gathered}
$$

Thus, the MBR (Equation 9) implies overestimation with halving and underestimation with doubling, which is what often has been found empirically (e.g., by Allan, 1978, and Eisler, 1975, for subjective duration). The midpoint $m\left(\mathrm{y}_{1}, \mathrm{y}_{2}\right)$ is always biased toward the upper end of the scale relative to the arithmetic mean, and this bias increases with the mean and the width of the middled interval (see Table 1).

In the two-dimensional case, the MBR (Equation 8) is approximately CB for smaller relative distances close to zero; in the midrange it is approximately euclidean, and for larger distances near one it tends toward the sup metric.

For one-dimensional psychophysical data, the bound $c^{*}$ can also be determined empirically, after expressing $\mathrm{d}(\mathrm{x}), \mathrm{h}(\mathrm{y})$, or $\mathrm{m}\left(\mathrm{y}_{1}, \mathrm{y}_{2}\right)$ in Equations 12-14 in terms of the original scale units. For example, for doubling, one obtains from Equation 12

$$
\mathrm{y}^{*}=2 \mathrm{x}^{*} \mathrm{c}^{* 2} /\left(\mathrm{c}^{* 2}+\mathrm{x}^{* 2}\right),
$$

and thus, 
Table 1

Bias Induced by MBR (9) Into Doubling, Halving, and Middling

\begin{tabular}{|c|c|c|c|c|c|c|c|c|c|c|c|c|}
\hline & .0 & .1 & .2 & .3 & .4 & .5 & .6 & .7 & .8 & .9 & 1.0 & \\
\hline .0 & .000 & .050 & . 101 & .154 & .209 & .268 & .333 & .408 & .500 & .627 & 1.0 & Halving (13) \\
\hline .1 & & .198 & .150 & .202 & .256 & .314 & .377 & .449 & .537 & .656 & 1.0 & \\
\hline .2 & & & .385 & .251 & .303 & .359 & .420 & .489 & .572 & .684 & 1.0 & \\
\hline .3 & & & & .550 & .351 & .405 & .463 & .529 & .607 & .712 & 1.0 & \\
\hline .4 & & & & & .690 & .451 & .507 & .569 & .642 & .739 & 1.0 & \\
\hline .5 & & & & & & .800 & .552 & .610 & .677 & .766 & 1.0 & Middling (14) \\
\hline .6 & & & & & & & .882 & .653 & .714 & .794 & 1.0 & \\
\hline .7 & & & & & & & & .940 & .756 & .824 & 1.0 & \\
\hline .8 & & & & & & & & & .976 & .859 & 1.0 & \\
\hline .9 & & & & & & & & & & .994 & 1.0 & \\
\hline 1.0 & & & & & & & & & & & 1.0 & Doubling (12) \\
\hline
\end{tabular}

Note-Boldface type indicates halving, and bold italic type indicates doubling; all other entries are results for middling. Examples: $d(.5)=.800<1.0 ; h(.5)=.268>.250 ; m(.2, .8)=.572>.500$.

$$
\mathrm{c}^{* 2}=\mathrm{y}^{*} \mathrm{x}^{* 2} /\left(2 \mathrm{x}^{*}-\mathrm{y}^{*}\right)
$$

However, since the denominator in Equation 16 is likely to be small, such estimates tend to be unreliable, so that it is probably best to obtain $\mathrm{c}^{*}$ by direct estimation, as Caelli et al. (1978) did. A reanalysis of some of their data suggests that the values predicted for doubling, middling, and halving in Equations 12-14 tend to be quite robust under variations of $\mathrm{c}^{*}$.

In terms of raw data, the MBR (Equation 8) can be written:

$$
\mathrm{d}_{\mathrm{ij}} *=\mathrm{c}^{* 2}\left(\mathrm{~d}_{\mathrm{ij} 1} *+\mathrm{d}_{\mathrm{ij} 2} *\right) /\left(\mathrm{c}^{* 2}+\mathrm{d}_{\mathrm{ij} 1} * \mathrm{~d}_{\mathrm{ij} 2} *\right) .
$$

\section{REFERENCES}

Allan, L. G. Comments on current ratio setting models for time perception. Perception \& Psychophysics, 1978, 24, 444-450.

Caelli, L., Hoffman, W., \& Lindman, H. Subjective Lorentz transformations and the perception of motion. Journal of the Optical Society of America, 1978, 68, 402-411.

EisLe r, H. Subjective duration and psychophysics. Psychological Review, 1975, 82, 429-450.

\section{NOTE}

1. These are Minkowski isometries, that is, in the simplest case, motions that preserve hyperbolas as unit disks.

(Received for publication March 8, 1982.) 